\title{
POLARIZATION MEASUREMENTS OF A STORAGE CELL TARGET
}

\author{
M.A. Ross, A.D. Roberts, T. Wise, and W. Haeberli \\ University of Wisconsin, Madison, Wisconsin 53706 \\ W.A. DeZarn, J. Doskow, H.O. Meyer, R.E. Pollock, B. von Przewoski, \\ T. Rinckel, and F. Sperisen \\ Indiana University Cyclotron Facility, Bloomington, Indiana 47408 \\ P.V. Pancella \\ Western Michigan University, Kalamazoo, Michigan 49008
}

A storage cell has been constructed for use as an internal target at the IUCF electroncooled storage ring (Cooler). We report here on nuclear polarization measurements of hydrogen, produced by an atomic beam source (ABS), in this storage cell. The results indicate a target polarization in excess of 0.70 for atoms in a single spin state.

The cell has a $1 \mathrm{~cm} \times 1 \mathrm{~cm}$ aperture to allow for passage of the circulating beam and is $25 \mathrm{~cm}$ in length. The cell wall is made from $5-\mu \mathrm{m}$ Teflon foil, which allows for the detection of low energy scattered particles. Polarized hydrogen (or deuterium) atoms enter the central region of the storage cell through an aluminum feed tube that is $1 \mathrm{~cm}$ in diameter and $13 \mathrm{~cm}$ long. In order to ensure long polarization lifetimes of atoms in the cell, the feed tube has been coated with Teflon. ${ }^{1}$

In preparation for experiments on the Cooler, polarization measurements using the storage cell target described above were performed at the UW Tandem accelerator using $\mathrm{p}+\mathrm{p}$ elastic scattering. At energies less than $8 \mathrm{MeV}$ and scattering angles near $\theta_{\mathrm{cm}}=90^{\circ}$, the spin correlation parameters $A_{y y}$ and $A_{x x}$ are both $-0.99 \pm 0.01$ and the analyzing power is $A_{y}=0$ (Ref. 2). Therefore, with vertical beam and target polarization, the differential cross section to a very good approximation becomes

$$
\sigma=\sigma_{0}\left(1-p_{b} p_{t}\right)
$$

where $p_{b}$ is the beam polarization and $p_{t}$ is the target polarization. The target polarization for spin up $(\uparrow)$ and spin down $(\downarrow)$ can easily be determined from the yields in the two beam polarization states

$$
p_{t}^{\uparrow}=\frac{1}{p_{b}} \frac{Y_{\downarrow \uparrow}-Y_{\uparrow \uparrow}}{Y_{\downarrow \uparrow}+Y_{\uparrow \uparrow}} \text { and } p_{t}^{\downarrow}=\frac{1}{p_{b}} \frac{Y_{\downarrow \downarrow}-Y_{\uparrow \downarrow}}{Y_{\downarrow \downarrow}+Y_{\uparrow \downarrow}} \text {. }
$$

For the yields in Eq. 2, the first arrow indicates the beam polarization and the second arrow is the target polarization. The beam polarization is measured separately in a polarimeter using $\mathrm{p}+{ }^{4} \mathrm{He}$ elastic scattering. ${ }^{3}$ Typically, the value is $p_{b}=0.88 \pm 0.01$.

Figure 1 shows the ABS, storage cell, and target chamber mounted at the high energy end of the tandem. Polarized 7.6-MeV protons travel through the storage cell into the beam polarimeter. Polarized hydrogen atoms from the ABS enter the storage cell through a feed tube mounted at an angle of $60^{\circ}$ with respect to the tandem beam line. Silicon strip 


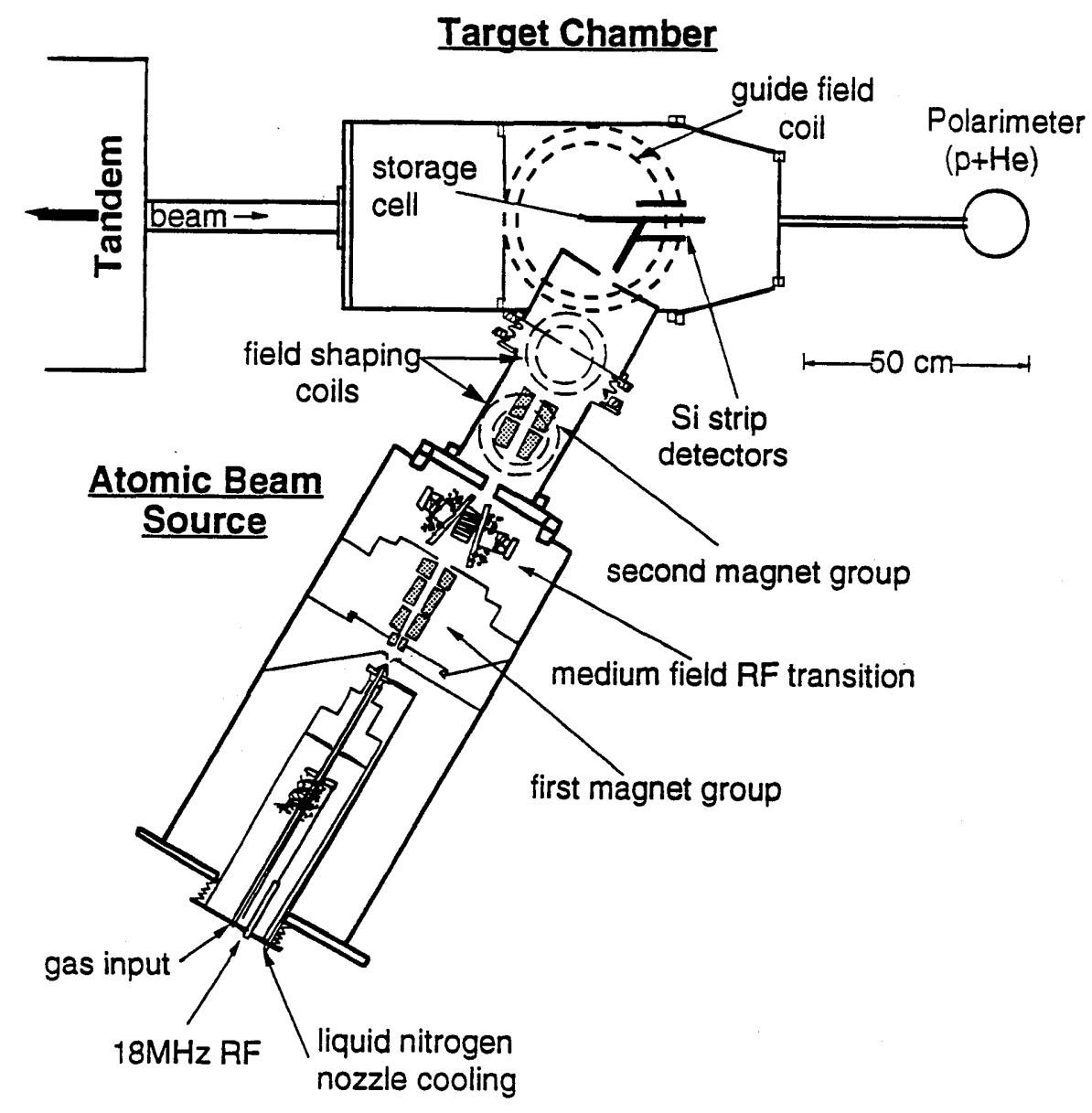

Figure 1. Layout of the Atomic Beam Source at the UW Tandem accelerator.

detectors located $5 \mathrm{~cm}$ from the center of the storage cell measure the energy and position of the scattered and recoil protons. Each detector has an active area of $4 \mathrm{~cm}$ by $6 \mathrm{~cm}$, is 1 - $\mathrm{mm}$ thick, and has 28 strips. A total of four detectors are used in these measurements. The azimuthal position of the detectors is chosen to avoid interference of the scattered protons with the feed tube assembly. As seen by the beam, the detectors are located in the upper-left and lower-right quadrants. Since the density of target atoms along the storage cell decreases linearly from the central region of the cell, the location of the detectors along the beam direction is chosen to give the maximum pp coincident rate.

An event is defined by a coincidence between the upper-left and lower-right detectors. For each event the energy and position signal of each detector that fired is recorded. The beam polarization is reversed every second; the target polarization is reversed after every run (approximately once an hour). Fig. 2 shows a plot of the energy in the upper-left detector $E_{L}$ vs. the energy in the lower-right detector $E_{R}$ for each beam spin state. The pp elastic events are clearly evident along the diagonal. The background is typically $4 \%$ of the total number of events and is determined from measurements with an empty cell. 

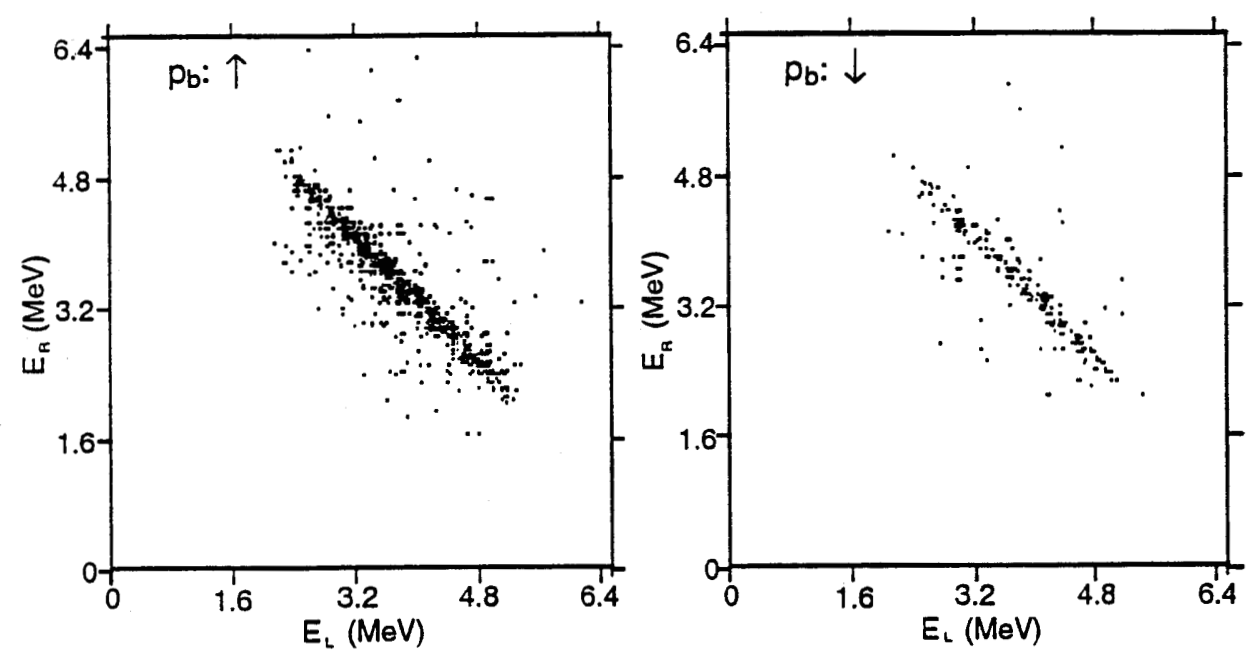

Figure 2. Energy in the upper-left detectors $\left(E_{L}\right)$ vs. energy in the lower-right detectors $\left(E_{R}\right)$. PP elastic events are along the diagonal. The direction of the target polarization is down.

The target polarization was first measured with the medium field RF transition (see Fig. 1) turned off. In this situation, atoms in both $m_{j}=+1 / 2$ spin states are focused by the ABS magnets and enter the storage cell. The guide field coils shown in Fig. 1 define the target polarization direction and produce a magnetic field of $1.5 \mathrm{mT}$. Under these conditions, the polarization of the atoms in the storage cell, assuming no polarization losses in the cell, is expected to be 0.485 . The measured value is $0.436 \pm 0.022$.

The target thickness, determined from the rates and the known $\mathrm{p}+\mathrm{p}$ cross section, was $2 \times 10^{13}$ atoms $/ \mathrm{cm}^{2}$. A new cell with a beam opening of $8 \mathrm{~mm}$ by $8 \mathrm{~mm}$ is currently under construction. With this new cell and improved vacuum conditions in the ABS, a target thickness of $7.2 \times 10^{13}$ atoms $/ \mathrm{cm}^{2}$ in the two spin states is expected.

When the medium field RF is turned on, a transition is made from the $m_{j}=+1 / 2$, $m_{i}=-1 / 2$ atoms to the $m_{j}=-1 / 2, m_{i}=-1 / 2$ atoms. The second magnet group (see Fig. 1) then defocuses the $m_{j}=-1 / 2$ atoms. This reduces the flux of atoms into the feed tube by about a factor of two, but increases the polarization of the atoms in the cell. Assuming no losses in the cell the polarization is

$$
p=\frac{1-\varepsilon a-(1-\varepsilon) R}{1+\varepsilon+(1-\varepsilon) R}
$$

where $a$ is the correction for the $1.5-\mathrm{mT}$ field over the cell, $(1-\varepsilon)$ is the efficiency of the medium field unit, and $R$ is the fraction of atoms in the cell with $m_{j}=-1 / 2$ that are not sufficiently defocused by the second magnet group. For a magnetic field of $1.5 \mathrm{mT}$, $a=0.03$. The efficiency of the medium field transition has been measured previously to be 
0.96 (Ref. 4). The value of $R$ is 0.052 , determined from transport calculations of the magnet assembly. The polarization with the medium field transition on is therefore expected to be 0.871 . The measured value is $0.722 \pm 0.021$. Table I summarizes all polarization values.

Table I. Polarization Results

\begin{tabular}{|c|c|c|c|c|}
\hline Medium Field & $p_{t}(\uparrow)$ & $p_{t}(\downarrow)$ & $\left|p_{t}\right|($ ave $)$ & $p_{\text {calc }}$ \\
\hline OFF & $0.415 \pm 0.032$ & $-0.457 \pm 0.031$ & $0.436 \pm 0.022$ & 0.485 \\
\hline ON & $0.701 \pm 0.029$ & $-0.743 \pm 0.030$ & $0.722 \pm 0.021$ & 0.871 \\
\hline
\end{tabular}

The results indicate that when the medium field transition is on we obtain a polarization that is $83 \%$ of the expected maximum. The reason for the $17 \%$ loss is not known at the present time. Inefficiency in the medium field unit or polarization losses in the cell are two possibilities. However, the polarization is large enough for experiments at the IUCF Cooler to begin later this year.

1. J.S. Price, Ph.D. Thesis, University of Wisconsin, 1993.

2. R.A. Arndt, L.D. Roper, R.L. Workman, and M.W. McNaughton, Phys. Rev. D 45, 3995 (1992); and the SAID database mentioned therein.

3. P. Schwandt, T.B. Clegg, and W. Haeberli, Nucl. Phys. A163, 432 (1971).

4. A.D. Roberts, P. Elmer, M.A. Ross, T. Wise, W. Haeberli, Nucl. Instrum. and Methods A322, 6 (1992). 